\title{
Dual-Color Fluorescence Cross-Correlation Spectroscopy for Monitoring the Kinetics of Enzyme-Catalyzed Reactions
}

\author{
Markus Rarbach, Ulrich Kettling, ${ }^{1}$ Andre Koltermann, and Manfred Eigen
}

Department of Biochemical Kinetics, Max Planck Institute for Biophysical Chemistry, Göttingen, Germany

Dual-color fluorescence correlation spectroscopy is a biophysical technique that enables precise and sensitive analyzes of molecular interactions. It is unique in its ability to analyze reactions in real time at nanomolar substrate concentrations and below, especially when applied to the monitoring of enzyme-catalyzed reactions. Furthermore, it offers a wide range of accessible reactions, restricted only by the prerequisite that a chemical bond or a physical interaction between two spectrally distinguishable fluorophores is established or broken. Recently, the optical setup of dual-color fluorescence correlation spectroscopy has been extended toward two-photon excitation, resulting in several advantages compared with standard excitation, such as lower fluorescence background, an even larger spectrum of potential fluorescence dyes to be used, as well as a more stable and simplified optical setup. So far, the method has been successfully employed to analyze the kinetics of nucleic acid and peptide modifications catalyzed by nucleases, polymerases, and proteases. @ 2001 Academic Press

Key Words: fluorescence correlation spectroscopy; dual-color cross-correlation spectroscopy; confocal fluorescence coincidence analysis; two-photon excitation; endonuclease; protease.

Fluorescence correlation spectroscopy (FCS) was introduced in the early 1970s (1-5). Confocal spectroscopy in general and FCS in particular open the possibility of observing processes on the single-molecule level. The range of FCS applications extends from photophysical dynamics of fluorescent dyes or proteins (6-8), conformational dynamics $(9,10)$, and interactions of macromolecules $(11,12)$ to biochemical kinetics $(13-15)$ to name only a few of numerous examples. FCS measure

\footnotetext{
${ }^{1}$ To whom correspondence should be addressed at Max Planck Institute for Biophysical Chemistry, Department 081, Am Fassberg, D-37077 Göttingen, Germany. E-mail: ukettli@gwdg.de.
}

ments are usually performed in an open confocal volumeelement vanishingly small compared with thetotal sample vol ume. Here diffusion coefficients and concentrations of the fluorescent species can be calculated from the correlation amplitudes and correlation times. While autocorrelation analysis provides the sensitivity of observing properties of single molecules the method, in some circumstances, lacks the specificity for detecting a particular fluorescent species one may be interested in, e.g., the substrate or the product of a biochemical reaction. More subtle changes of one fluorescent species are sometimes obscured by others. This inherent limitation of single-color methods becomes more significant if short measurement times with excellent signal-to-noise ratios are to be achieved as it is a prerequisite for high-throughput screening applications. Dual-color approaches can provide this specificity while preserving the high sensitivity of FCS.

Application of dual-color fluorescence cross-correlation in the biosciences was first suggested by Eigen and Rigler in 1994 (16). The first experimental demonstration of cross-correlation was reported by Schwille et al. (17) with measurements of the hybridization of two ol igonucleotides labeled with different fluorescent dyes. Rigler et al. (18) applied the concept to the detection of amplified polymerase chain reaction products employing labeled primers for both strands. Like DNADNA interactions protein- protein interactions also can be detected, as demonstrated only recently by Bieschke et al. (12) with the sensitive measurement of prionprotein aggregations. Direct observation of enzymatic processes has been put forward by Kettling et al. with on-line measurements of the enzymatic cleavage of a DNA substrate by EcoRI endonuclease (13) and by Koltermann et al. with the enzymatic degradation of a 
peptide by the protease subtilisin (19). Recent developments of dual-color approaches extend their applicability from purely explorative subjects to sensitive detection in high-throughput-screening applications (20). New and promising photophysical detection principles (21) as well as new data processing methods complementing correlation analysis (22-24) have been developed.

\section{DUAL-COLOR CONFOCAL FLUORESCENCE SPECTROSCOPY: OPTICAL SETUP AND ADJUSTMENT}

Most technical realizations of systems detecting fluorescence fluctuations on the molecular level employ a confocal optical setup. The basic principle here is the epi-illumination of a measurement volume of several $100-n m$ in diameter with a high-aperture microscope objective. By virtue of their high spatial, temporal, and spectral stability, lasers are used for illumination of the focal spot. By placing a confocal pinhole in the image plane of the objective the axial dimensions of the measurement volume can be restricted to few micrometers in length. The resulting open detection volumeis correspondingly of about $1 \mathrm{fl}$ in size. Fluorescence photons emanating from this small open detection volume are recorded by a sensitive detector, e.g., avalanche photodiodes. Excitation and detection wavelengths have to be separated by appropriately chosen dichroic mirrors and filters.

The optical setup resembles single-color systems but is somewhat complicated by the necessity to align the excitation beam paths and detection optics, on the one hand, and discrimination between two excitation and two emission wavelengths by a properly chosen set of dichroic mirrors and filters, on the other hand. The experimental realization first proposed by Schwille et al. in 1997 (17) is sketched in Fig. 1. Two coaxial laser beams originating either from two separate lasers superimposed by a dichroic beam combiner (17) or from a multiline laser as demonstrated by Winkler et al. in 1999 (20) are reflected by a dichroic mirror to the back aperture of the microscope objective. The fluorescence emission from the focal spot is collected by the highaperture objective, traverses the dichroic mirror, and is focused to the pinhole in the image plane with a diameter of 30-70 $\mu \mathrm{m}$. After passing the pinhole the two emission wavelengths are separated by a dichroic beam splitter and focused to separate detectors. Any of the dichroic mirrors may be combined with suitable optical filters to achieve proper spectral separation between the different spectral windows of excitation and emission. The optical properties of a system of filters

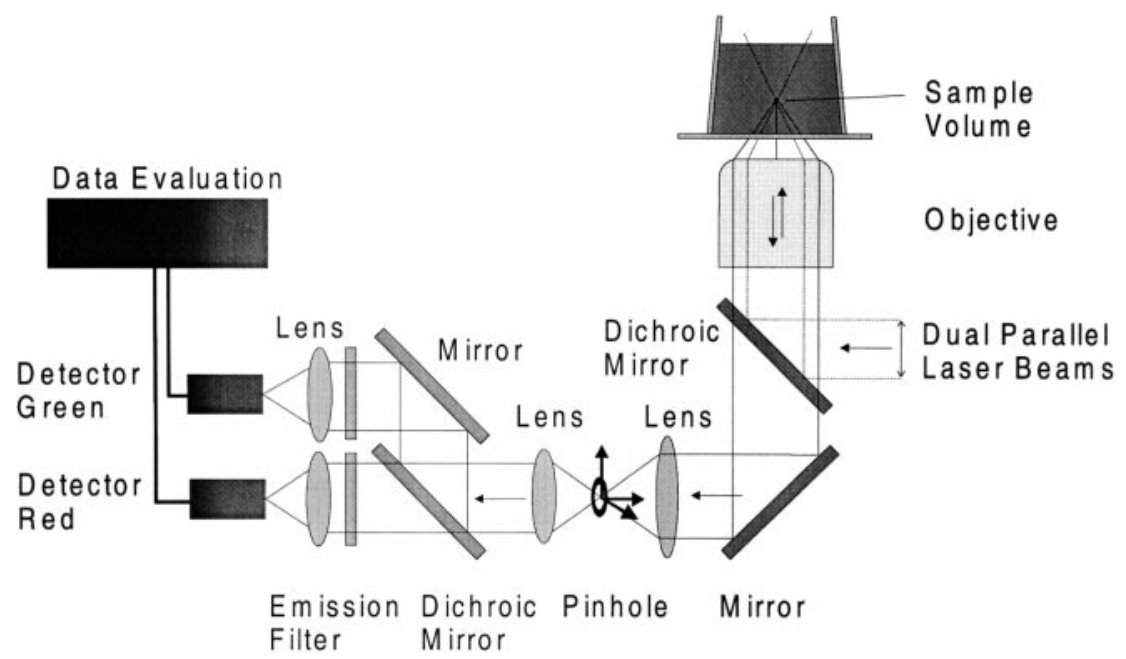

FIG. 1. Dual-color fluorescence cross-correlation spectroscopy setup. Two parallel laser beams are focused by a microscope objective on the sample volume. This results in two superimposed focal spots, forming a confocal volume element in the femtoliter range. The fluorescence light that is collected by the same microscope objective is separated from the excitation light by a dichroic mirror and focused onto a pinhole by a lens. The pinhole, with an adjustable diameter, is located in the image plane of this lens and its position can be adjusted precisely in the $x-y-z$ axes. Fluorescence emission is parallelized, separated by a dichroic mirror into green and red fractions, and refocused on two avalanche photo diodes. Reproduced with permission from U. Kettling, A. Koltermann, P. Schwille, and M. Eigen, (1998) Proc. Natl. Acad. Sci. USA 95, 1416-1420. Copyright 1998 National Academy of Sciences, U.S.A. 
and mirrors employed for detection of cross-correlation of a "green" and a "red" fluorescent dye are depicted in Fig. 2 (the terms "green" and "red" are chosen for simplicity; in dual-color applications dye combinations emitting in the green and red spectral regions are often chosen). Systems employing two pinholes, i.e., one for each detection channel, have also been implemented. This solution is technically more complex but allows for correction of wavelength-dependent effects such as different sizes of the confocal volume elements for the different excitation wavelengths.

Adjusting the optical system is of vital importance. While the basic adjustment of the system of lenses, mirrors, and filters is usually done once when installing the system, the positions of the pinholes in all three spatial dimensions have to be adjusted routinely (usually every day) but inevitably when dichroic mirrors

(A)

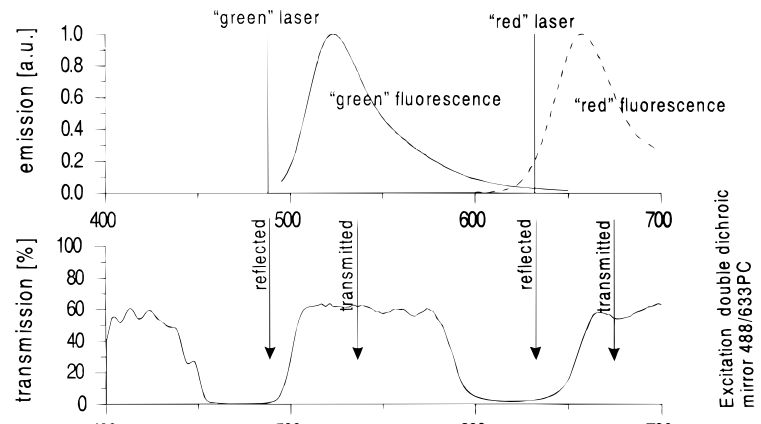

(C)

(D)
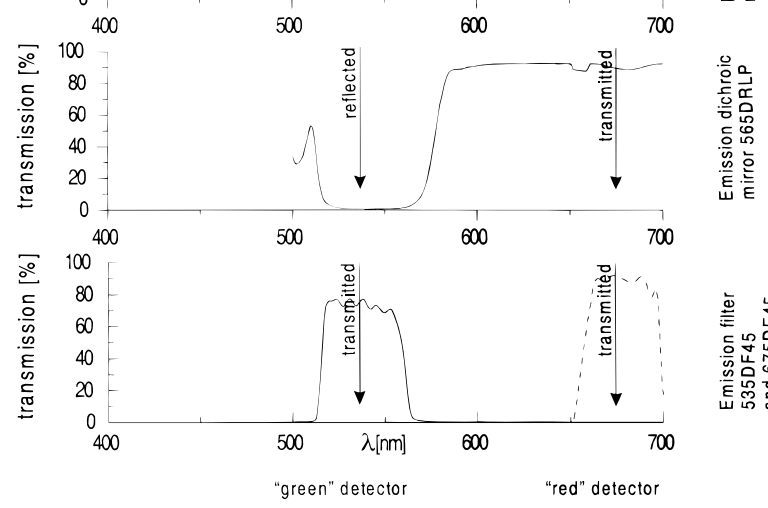

FIG. 2. Spectral separation of excitation and emission wavelengths (A) E mission of the 488-nm "green" laser line and the 632-nm "red" laser line and emission spectra of the "green" dye RhG and the "red" dye Cy5. (B) Wavelength selection by the excitation dual dichroic mirror. The two laser lines are reflected by the mirror from the laser sources to the back aperture of the objective while the fluorescence emission from the sample traverses the mirror. (C) Selection of the two fluorescence wavebands. While the emission of the "green" dye is reflected by the emission dichroic mirror to the "green" detector, "red" fluorescence passes the mirror to the "red detector." (D) Absorption filters for residual scattered laser emission. The emission and excitation dual dichroic will usually not block backscattered laser light efficiently enough. The separate emission filters therefore re move residual laser emission. have been changed to measure at different wavelength combinations. Adjustment is commonly done with solutions of free fluorescent dyes that are to be used in the experiments. Since the diffusion constants of freely diffusing dye molecules are known from the literature, proper adjustment of the system can be checked separately for each color by evaluating autocorrelation curves. However, to detect molecules at both colors simultaneously, as is necessary for cross-correlation analyses, it is vital that the open detection volumes at both wavelengths are identical. Obviously the most simple and meaningful routine checks are measurements with pure solutions of double-labeled molecules with known cross-correlation amplitudes. If these are not available, free fluorescent dyes might serve a similar purpose. As suggested by Schwille et al. (17) overlap of the two excitation volumes can be checked with a fluorescent dye of similar absorption cross section for the two excitation wavelengths. This is made possible by the the fact that most of thelong-wavel ength dyes havenonzero absorption cross sections at wavelengths well below their absorption maxima. If thecorrelation results measured for both excitation wavelengths without changing the detection optics are similar within $10 \%$, a reasonable overlap of the focal spots is given. In a similar procedure proper adjustment of the detection beam paths of both channels can be checked employing one dye emitting at both detection wavelengths. For this purpose short-wavelength dyes are used that commonly show nonzero emission at wavelengths considerably larger than their emission maximum. The short-wavelength dye is excited by the short-wavelength laser only and emission is detected in both channels. Then the accuracy by which the two autocorrelations and the cross-correlation resemble each other is a direct measure for the superimposition of the two detection volumes.

Apart from these purely instrumental requirements for cross-correlation spectroscopy several other points haveto beaddressed when designing a particular assay. First, a careful selection of the fluorescent dyes to be employed is essential. General properties of fluorophores to consider in any confocal spectroscopic application are photostabilities and quantum yields of the dye molecules, to accomplish good signal-to-noise ratios and consequently short measurement times. Rhodamine and cyanine dyes are usually a proper choice to start with. Parameters that are dual-color system-specific are the spectral separation of excitation and emission maxima and the cross-talk between the two emission spectra. As al ready pointed out longer-wavelength dyes have usually nonzero excitation cross sections at shorter wavelengths. This excitation cross-talk, i.e., both fluorescent dyes are excited by the same laser line, 
is less complicated to handle than emission cross-talk, where photons emitted by the same fluorescent dye are detected in both channels. Obviously, the former does not produce false positive cross-correlation while the latter does. Some cross-talk between the channels is inevitable but appropriate checks have to be performed and taken into account when interpreting the results.

Fluorescence quenching might be another issue in some applications. For instance it is known that rhodamine dyes can bequenched by guanineresidues in DNA substrates. Thequenching of the dye and concomitantly the fluorescence count rate might change in the course of a biochemical reaction, e.g., when exonucleolytically digesting a rhodamine-labeled DNA substrate. In first approximation this will not change the results of the correlation. For more precise analyses, this can be accounted for by multiparameter fitting of auto- and cross-correlation data in parallel using Eq. [5] (see be(ow). Additionally, the quenching observed can be dependent on the base sequence of the DNA substrateand caution might be advised in extrapolating experiences from one assay to another. Having considered all these points in designing the assay substrates, conditions in the confocal setup have to be determined, most importantly the concentration range at which the fluorescent substrate can be used and the excitation power to be chosen. This is essentially an experimental task. Fixing the excitation power involves a trade-off between photostability of the dyes, on the one hand, and good signalto-noise ratios or short measurement times, on the other hand. Generally, similar count rates for both fluorescent dyes is the aim. Determination of appropriate assay concentrations has to consider some more aspects. At relatively low particle numbers only few molecules traverse the focal spot in a given timeand analysis might be rather time consuming, whereas at relatively high occupation numbers the relative fluorescence fluctuations become smaller, rendering any confocal method insensitive. Therefore, the appropriate assay concentration is critically dependent on the aim of the measurement and the underlying knowledge. If little is known about the molecular species observed during the course of a reaction, e.g., in terms of diffusion times, quenching characteristics, moderate concentrations may be a good choice (e.g., occupation numbers between about 1 and 5). If, on the other hand, virtually everything is known about the assay except one particular parameter, then much higher concentrations can work well. For a more detailed discussions of the physical aspects of cross-correlation al gorithms and analyses see Schwille (25).

\section{TWO-PHOTON EXCITATION METHOD}

Since the technical task of aligning the two confocal laser spots to one identical measurement volume and ensuring stability of this setup is all but simple, one might think of two fluorescent dyes excited at the same wavelength but emitting at two spectrally separated wavelengths. Unfortunately, finding these two dyes with extremely different Stokes shifts proves to be a difficult task and no such combination has been proposed yet. A different solution to the problem has recently been proposed by Heinze et al. (21) by using twophoton excitation of fluorescent dyes.

The method makes use of the fact that fluorescent dyes with absorption maxima in the near-UV and visible spectral regions can be excited by quasi-simultaneous absorption (within $10^{-15} \mathrm{~s}$ ) of two or more photons from a near-IR laser, an effect generally refered to as two-photon or multiphoton excitation (26-28). Besides the crucial fact that in this way fluorophores emitting in different spectral regions can be excited by the same IR laser source, this solution has some more inherent advantages. The two-photon absorption process is nonlinear (formally it is of second order with respect to the radiative flux density). Accordingly the two-photon excitation probability declines with $z^{-4}$ in a distance $z$ from the focal plane. The pinhole needed in conventional confocal spectroscopy to constrain the focal volume in the $z$ direction is ther efore of minor importance in this application and may be omitted completely. This is obviously an attractive feature from the technical point of view and simplifies theadjustment of the setup. Another aspect arises in the context of measurements in biological samples such as measurements in theinterior of single cells or in tissues. Measurements at short wavelengths often suffer from intrinsic fluorescence of the sample itself, which complicates the measurement and the interpretation of the results. Two-photon excitation will considerably reduce similar problems. Onephoton Stokes fluorescence, if there is any at all, will be emitted at even larger wavel engths then the I R excitation and therefore will not interfere with the twophoton anti-Stokes fluorescence emission wavebands. Given the high radiative flux density required for FCS measurements decreased photodestruction of sample is another advantage of two-photon excitation. First, absorption cross sections in biological samples are considerably smaller in the IR than for visible irradiation; second, any two-photon excitation is restricted to the small focal volume since the excitation probability is of second order with respect to the radiative flux density and, therefore, virtually zero outside. 


\section{CROSS-CORRELATION AND COINCIDENCE ANALYSIS}

The primary variable measured in confocal fluorescence spectroscopy is the fluctuation of the fluorescence signal arising from single fluorescent molecules in the tiny focal measurement volume. Thefluctuations either may arise from the passing of molecules into and out of the open volume element or may be due to intrinsic molecular dynamics such as rotation, intersystem crossing to the triplet state, or other photophysical phenomena. The amplitude of fluctuations from any single fluorescent molecule is inversely related to the occupation number, i.e., the mean number of fluorescent molecules within the detection volume. Therefore, confocal fluorescence spectroscopy has the somewhat peculiar feature that concentrations of the substance to be measured are restricted to relatively low values, usually with an optimum at occupation numbers between 1 and 100. At occupation numbers well below 1, the same measurement precision needs drastically increased measurement time since most of the time there is no signal to be evaluated. On the other hand, at considerably higher occupation numbers the relative fluorescence shift due to single-molecule fluctuations becomes too small to be detected and evaluated accurately. In general, confocal fluorescence spectroscopy works well in the pico- to nanomolar range. For example, with a detection volume of $1 \mathrm{fl}$ an occupation number of approximately 1 is to be expected at a concentration of $1 \mathrm{nM}$. All confocal fluorescence spectroscopic methods evaluate such fluorescence fluctuations but differ in the al gorithm used to extract certain molecular properties. Which method is suitable to address a certain experimental task has to be decided for any particular case independently.

FCS, the most widespread and best known confocal spectroscopic method, uses correlation analysis to extract the temporal correlation of the signal and thereby the underlying time constants of fluorescence fluctuations. Assuming that diffusion is the sole source of fluctuation, this translates to mean occupation numbers and occupation times of the focal volume, which in turn may be expressed as diffusion constants and concentrations of the fluorescent molecular species in the system. However, other fluctuation sources such as conversion to triplet state can be extracted additionally. The normalized fluctuation correlation function $\mathrm{G}$ is given by

$\mathrm{G}_{\mathrm{ij}}(\tau)=\frac{\left\langle\delta \mathrm{F}_{\mathrm{i}}(\mathrm{t}) \delta \mathrm{F}_{\mathrm{j}}(\mathrm{t}+\tau)\right\rangle}{\left\langle\mathrm{F}_{\mathrm{i}}(\mathrm{t})\right\rangle\left\langle\mathrm{F}_{\mathrm{j}}(\mathrm{t})\right\rangle}=\frac{\left\langle\mathrm{F}_{\mathrm{i}}(\mathrm{t}) \mathrm{F}_{\mathrm{j}}(\mathrm{t}+\tau)\right\rangle}{\left\langle\mathrm{F}_{\mathrm{i}}(\mathrm{t})\right\rangle\left\langle\mathrm{F}_{\mathrm{j}}(\mathrm{t})\right\rangle}-1$,

where the fluorescence fluctuation $\delta \mathrm{F}$ is defined as the difference of the instantaneous fluorescence from the temporal mean, $\delta \mathrm{F}=\mathrm{F}(\mathrm{t})-\langle\mathrm{F}(\mathrm{t})\rangle$ and $\mathrm{i}, \mathrm{j} \in(\mathrm{r}, \mathrm{g})$ are the red and the green detection channels. Taking $\mathrm{i}=\mathrm{j}$ then leads to the autocorlation functions $G_{r r}$ and $G_{g g}$, while $i \neq j$ gives the cross-correlation function $G_{g r}$ for the two fluorescent species. Taking into account the characteristics of the excitation and detection optics a functional relation between the correlation function and the occupation times for translational diffusion for a single fluorescent species can be found (29):

$$
\begin{aligned}
\mathrm{G}_{\mathrm{ij}}(\tau) & =\mathrm{G}_{\mathrm{ij}}(0)\left(1+\frac{\tau}{\tau_{\text {Diff,ij }}}\right)^{-1}\left(1+\frac{\mathrm{r}_{0}^{2} \tau}{\mathrm{z}_{0}^{2} \tau_{\text {Diff, }, \mathrm{ij}}}\right)^{-\frac{1}{2}} \\
& =\mathrm{G}_{\mathrm{ij}}(0) \mathrm{Diff}_{\mathrm{ij}} .
\end{aligned}
$$

Here $r_{0}$ and $z_{0}$ are the radial and axial dimensions of the focal spot, $\tau_{\text {Diff }}$ is the characteristic diffusion time of the fluorescent molecules, and $\mathrm{G}(0)$ is the correlation amplitude. For systems with several fluorescent species the total correlation function may be expressed as a sum of independent correlations,

$$
G_{i j}(\tau)=G_{i j}(0) \cdot \frac{\sum_{k} Y_{k} \eta_{i j, k}^{2} D i f f_{i j, k}}{\left(\sum_{k} Y_{k} \eta_{i j, k}\right)^{2}},
$$

where $Y_{k}$ are the molar fractions of the $k$ fluorescent species and consequently $\sum_{k} Y_{k}=1$. With the assumption of constant fluorescence yield $\eta$ for all fluorescent species Eq. [3] then simplifies to

$$
\mathrm{G}_{\mathrm{ij}}(\tau)=\mathrm{G}_{\mathrm{ij}}(0) \cdot \sum_{\mathrm{k}} \mathrm{Y}_{\mathrm{k}} \mathrm{Diff}_{\mathrm{ij}, \mathrm{k}} \cdot
$$

Fluorescence fluctuations originating from intramolecular processes like intersystem crossing of fluorophores to the triplet state at high radiative flux density have to be taken into account additionally. The correlation function then has to be corrected for the fraction of dye molecules in triplet state $\mathrm{T}$ :

$$
G_{i j}(\tau)=G_{i j}(0) \cdot \frac{1}{(1-T)}\left(1-T+T e^{\left(-\tau / \tau_{T}\right)}\right) \sum_{k} Y_{k} D_{i f f} f_{i, k} .
$$

All parameters $\left(\tau_{\text {Diff, },}, G_{(0)}, Y_{k}, T\right.$, and $\left.\tau_{\mathrm{T}}\right)$ are commonly estimated from fitting Eq. [5] to the measured correlation function. Concentration and diffusion constants estimated from the autocorrelation functions are then given by 


$$
\begin{gathered}
c_{r r, k}=Y_{k} /\left(G_{r r}(0) V_{\text {eff }}\right), \quad c_{g g, k}=Y_{k} /\left(G_{g g}(0) V_{\text {eff }}\right), \\
\tau_{D i f f, i j, k}=r_{0, i j}^{2} / 4 D_{k} .
\end{gathered}
$$

The effectivemeasurement volume $V_{\text {eff,ij }}=\left(\pi^{3 / 2} r_{0}^{2} z_{0}\right.$ and structure parameters $\left(z_{0} / r_{0}\right)$ are generally and conveniently estimated from calibration measurements with free fluorescent dyes of known concentration and diffusion constants. The relative amplitude of the cross-correlation function is directly proportional to the occupation number of the focal spot since for cross-correlation the denominator of Eq. [1] encompasses all fluorescent molecules (all auto- and cross-correlating species) while the numerator is a function of the cross-correlating species only. With the assumption of identical detection volumes $\mathrm{V}_{\text {eff }}$ for all correlations the concentration of the cross-correlating species is then given by

$$
C_{g r}=G_{g r}(0) /\left(V_{e f f} G_{g g}(0) G_{r r}(0)\right)
$$

To exemplify the advantages of cross-correlation consider the reaction sketched in Fig. 3 assuming the enzymatic cleavage of a macromolecular substrate. In scheme $A$ the molecule carries only a single label. The number of fluorescent particles will not change in the course of the reaction, whereas the molecular weight and therefore the diffusion time of the product will. With the Stokes-Einstein relation

$$
\mathrm{D}=\frac{\mathrm{kT}}{6 \pi \eta \mathrm{R}_{\mathrm{h}}}
$$

and the assumption that in the first approximation the hydrodynamic radius $R_{h}$ of a molecule may be assumed to be proportional to the cubic root of its molecular weight $M$, i.e., $R_{h} \approx \sqrt[3]{M}$, Eq. [6] may be written

$$
\tau_{\text {Diff }}=\pi \frac{3}{2} \frac{r_{0}^{2} \eta}{\mathrm{kT}} \sqrt[3]{\mathrm{M}}
$$

The diffusion time of the product with half of the molecular weight of the substrate may therefore be expected to be only $20 \%$ smaller than the diffusion time of the substrate. In scheme B the substrate molecule carries two identical labels. For the diffusion constant thesame applies as in scheme $A$ but the particle number will change by a factor of 2 in the course of the reaction. This is surely an advance in comparison to the conditions of scheme A. But taking into account nonideal conditions encountered in real measurements some more subtle changes in the reaction may be difficult to detect if the utmost change we can expect is doubling of the particle number. In scheme $C$ the mol ecule carries two different labels. Since the cross-correlation amplitude is directly proportional to the number of cross-correlating molecules, here the total dynamic range of the correlation amplitude can be used for data evaluation. Under ideal experimental conditions this may encompass two orders of magnitude, opening thereby the possibility of measuring enzyme activities that lead only to very small changes in substrate or product concentrations.

It may be only briefly mentioned here that in the experimental setup nonideal conditions will be encountered, e.g., differing effective measurement volumes at the different wavelengths, cross-talk between the fluorescent dyes, and incomplete overlap of the focal spots, which are not covered by the above calculus. Again for a thorough discussion Schwille (25) may be advised.

Evaluation of thecorrelation function gives estimates for the concentration and the diffusion times of the fluorescent species. While both parameters are of significance in many explorative experimental protocols the situation is different in screening applications. Here often only concentrations of thefluorescent species have to be measured and short measurement times are important when large libraries areto be screened. Winkler et al . (20) have proposed a simplified analysis for these applications termed confocal fluorescence coincidence analysis (CFCA), which allows for on-line evaluation of the concentrations of cross-correlating particles in the sample. This is in contrast to FCS, where the fitting procedure usually is not performed concurrent to the measurement. The coincidence $K$ is defined as

$$
K=\frac{n \sum_{n} N_{i} N_{j}}{\sum_{n} N_{i} \sum_{n} N_{j}}
$$

where $\mathrm{N}_{\mathrm{ij}}$ are photon count numbers for the two emission wavelengths sampled in a time interval $\Delta t$ and $\left(N_{i, j}=\int_{\Delta t} F_{i, j}(t) d t\right)$ and $n$ is thenumber of timeintervals of length $\Delta$ t sampled. The coincidence is formally identical to the, commonly nonexistent, zeroth channel of the cross-correlation and takes advantage of the fact that the cross-correlations are inherently shot-noise-free in contrast to the autocorrelations. Furthermore it is advantageous that the coincidence value determined is insensitive to movement of the sample. The lower boundary for analysis times in confocal setups is given by the fact that a statistically meaningful number of molecules has to be detected. The number of molecules diffusing through the focal spot is proportional to the concentration and the diffusion constant of the fluorescent species (16). A lower threshold of the measurement 
time for a required signal-to-noise ratio is produced by the limited concentration range for confocal measurements (as pointed out above) and the fact that the diffusion constant cannot usually be raised significantly. However, without changing the occupation number the number of molecules detected can be increased by applying a relative movement of focal volume and sample, a procedure usually termed "scanning." Using CFCA and implementing relative movement of thesampleWinkler et al. (20) demonstrated that on-lineevaluation of fluorescence signals with analysis times of 100 $\mathrm{ms}$ and shorter enables unambiguous (1.4\% overlap of positive and negative samples) measurements of enzymatic activity in screening applications.

As an alternative to correlation analysis in FCS, single-col or histogram analyses have been established independently as fluorescence intensity distribution analysis (FIDA) (22) and photon counting histogram $(\mathrm{PCH})$ analysis (23). FIDA has recently been extended to two-color applications by Kask et al. (24). Basically the method employs a histogram presentation of the fluorescence emission sampled in fixed time intervals of length $\Delta \mathrm{t}$. Deconvolution of the histogram gives information about the intensity distribution of the emission and thereby of the molecular composition of the sample. The complete mathematical calculus of the method is rather complex and not within the scope of this article. The essential point to keep in mind is that histogram analysis can deduce particle numbers and molecular brightness values for different fluorescent species, contrasting and complementing FCS, where particle numbers and diffusion times can be calculated. Therefore, suitable targets for this method are assays where only minor changes in the diffusion characteristics are to be expected. As an example, molecular brightness is the method of choice to determine the fraction of ligandbound to unbound receptor molecules integrated into a lipid vesicleand, thereby, to enablethedeterminination of affinity constants of ligands or inhibition efficiency of potential receptor antagonists (24).

\section{EXAMPLE: ENDONUCLEASE KINETICS}

The first example employing the particular features of dual-color FCS for the analysis of enzyme kinetics was the investigation of endonuclease-catalyzed DNA digestion. This study was done in the authors' laboratory using the class II restriction enzyme EcoRI as a model enzyme (13). Endonucleases in general and class II restriction enzymes in particular are well known enzymes, whose catalytic mechanisms and kinetics have been thoroughly investigated. Consequently, there exists an arsenal of methods for analyzing the catalytic activity of restriction endonucleases. Many of them are very sensitive, especially when employing radioactive labels and separation techniques such as electrophoresis. Sensitivity is important, because the substrate recognition of restriction endonucleases is extraordinarily specific, corresponding to $\mathrm{K}_{\mathrm{m}}$ values in the nanomolar range. However, all but a few of these techniques are inevitably off-line. On-line methods, on the other hand, that enable monitoring of endonucleolytic reactions in real time in a homogeneous assay format usually suffer from a rather low sensitivity.

For example, Waters and Connolly (30) employ the increased UV absorbance of single-stranded DNA compared with double-stranded DNA (hyperchromic effect). Short oligonucleotides (6- 8 nucleotides) resulting from endonucleolytic cleavage will dissociate spontaneously and can be detected on-line, but DNA concentrations in the micromolar range are required for the procedure. Ghosh et al. (31) employ fluorescence resonance energy transfer (FRET) in labeled 16- to 19-base oligonucleotides for detection of the substrate cleavage. The method shows sensitivity in the nanomolar range but suffers from the fact that the fluorescent labels have to be in close vicinity to the cleavage site to show the desired resonance energy transfer. Interactions between the labels and the enzyme can therefore not be ruled out. In fact the results show that the shortest oligonucleotide tested shows higher affinity to the enzyme, which Ghosh et al. (31) attributed to hydrophobic interactions between the fluorescent labels and the enzyme. Halford and J ohnson (32) follow the decrease in fluorescence of ethidium bromide released from a degraded double-stranded DNA substrate. A sensitivity of the assay in the nanomolar range is reported. Although any effect of the intercalated ethidium bromide on the enzymatic process is ruled out by the authors, interactions between the dye and the enzyme and distortions of the DNA structure by the dye cannot be excluded generally. The combination of both necessities, i.e., real-time analyses in homogeneous formats combined with sensitivities at and below the $K_{m}$ of restriction enzymes, is achieved only by dual-color confocal fluorescence techniques such as dual-color FCS.

EcoRI recognizes as a dimer specifically the palindromic recognition sequence GAATTC in a DNA double strand and catalyzes hydrolysis of the phosphodiester bond between the guanosine and the first adenosine monomer in each of both strands (33). To enable analysis of cleavage by dual-color FCS, in the above-mentioned study a linear, double-stranded, 66-bp-long DNA substrate containing the EcoRI recognition site was 
synthesized and labeled at the two respective $5^{\prime}$ ends with spectrally distinguishablefluorophores. Thefluorophores chosen were Rhodamine Green (RhG, MolecuIar Probes, Eugene, OR), referred to as the green label and excited with the blue 488-nm line of an $\mathrm{Ar}^{+}$laser, and Cy5 (Amersham Pharmacia), referred to as the red label and excited with the red 633-nm line of a $\mathrm{HeNe}$ laser. The 66-bp DNA molecule can be expected to have
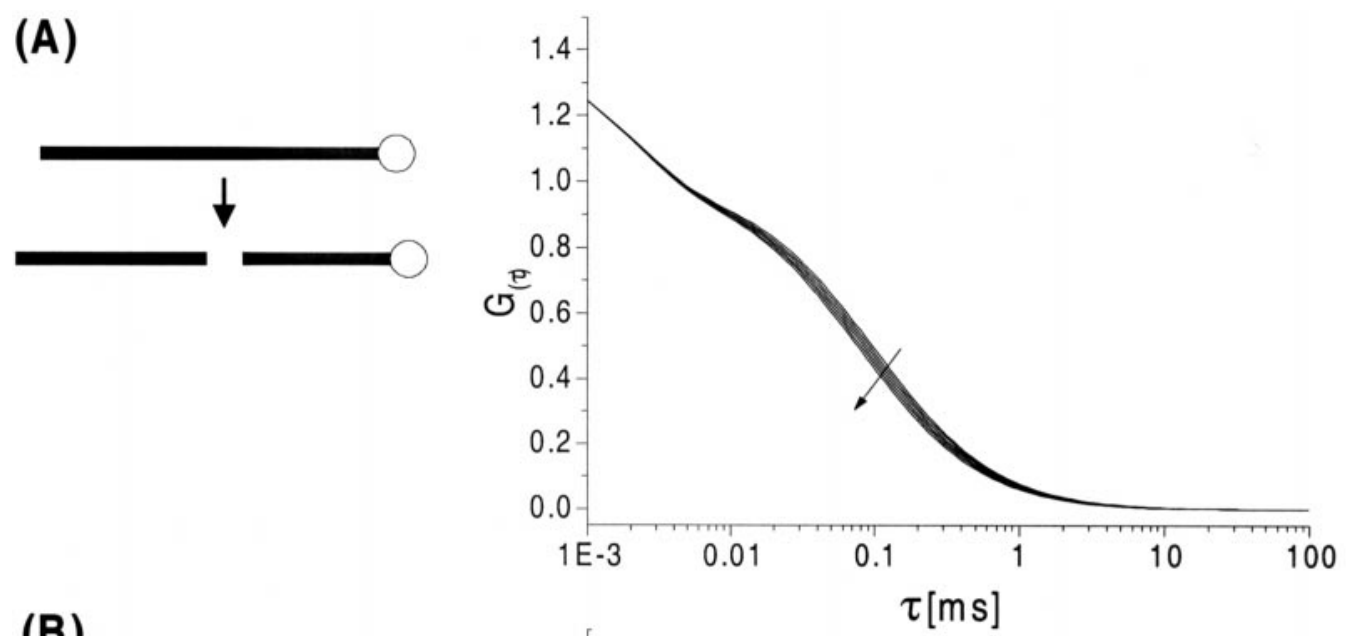

(B)
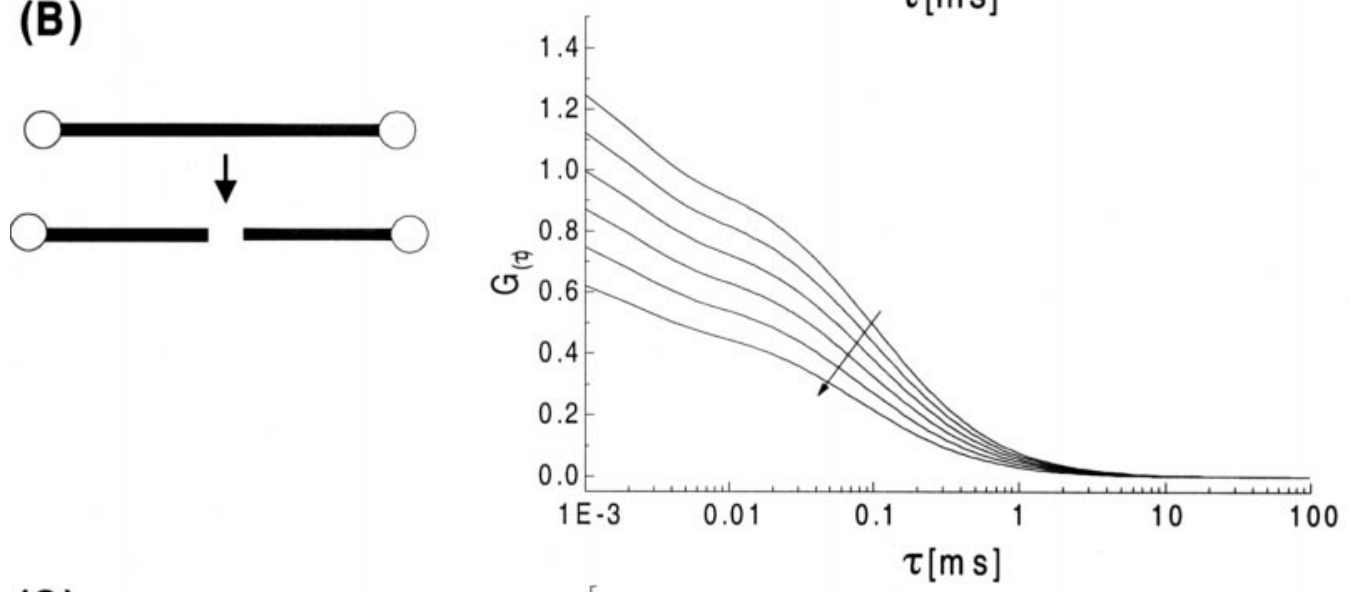

(C)
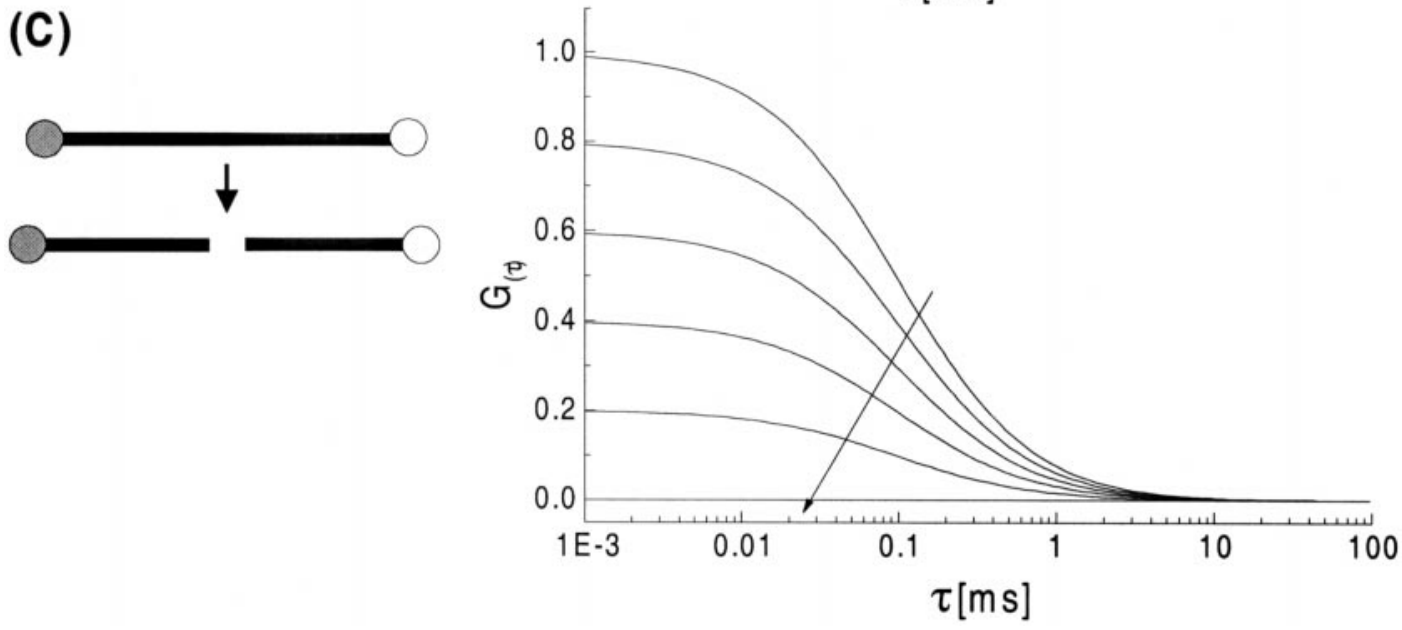

FIG. 3. Comparison of single- and dual-col or measurements for the analysis of cleavage reactions. Correlation curves calculated from Eqs. [2], [3], and [5]. Scheme A: single-label substrate; scheme B: double-label single-color substrate; scheme C: double-label dual-color substrate. Arrows indicate reaction progress. For explanation, see text. 
a linear structure in solution with the labels attached to opposite ends. When comparing the sizes of the substrate molecule and the enzyme dimer when drawn in scale (see Fig. 4), it is obvious that the distance between the cleavage site and the fluorophores avoids any interaction between enzymeand labels as is typical for other artificial substrates. This particular feature of dualcol or FCS, i.e., the general freedomtoattach thefluorescent labels anywhere appropriate at the molecule to be detected, is one of the striking advantages compared with assay principles based on theinteraction of fluorophores with each other (e.g., fluorescence resonance energy transfer) or with other molecule portions (e.g., quencher groups) (31). By applying different fractions of labeled to unlabeled substrate at constant overall substrate concentrations, it has been experimentally verified that the labels attached at the ends of the DNA molecule do not interfere with the enzyme's catalytic action. Moreover, the labeled substrate can serve as a one-to-one indicator for cleavage of unlabeled DNA, allowing measurement at a much broader concentration range than appropriate when applying only the fluorescent substrate (13).

Hydrolysis of the DNA molecule followed by release of the two fragments from the enzyme dimer interrupts the linkage between the two fluorophores and results in loss of the cross-correlation signal. To monitor the time course of this reaction in solution, the fluorescence signals are measured continuously, and cross-correlation data analysis (see Eq. 1) is carried out at constant intervals. From the amplitude $\mathrm{G}_{\mathrm{gr}}(0)$ of the cross-correlation curves (shown in Fig. 5), which are proportional to the fraction of double-labeled molecules in solution, the decreasing concentration of nonhydrolyzed DNA substrate over time can be calculated. Using this data evaluation, the kinetics of EcoRI cleavage have been analyzed at substrate concentrations between 1 and

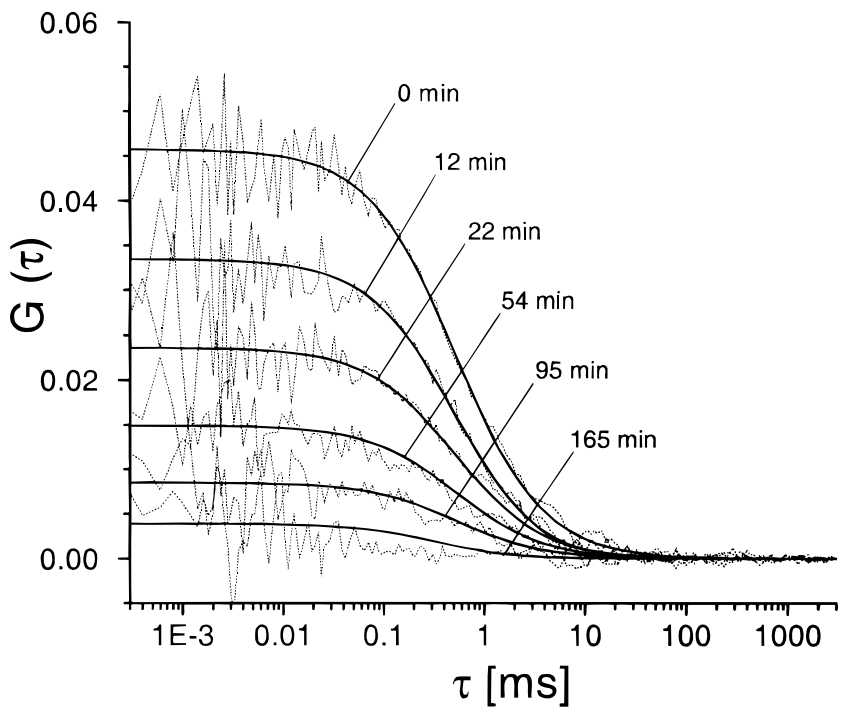

FIG. 5. Cross-correlation curves at different time points during endonucleolytic cleavage of a double-labeled oligonucleotide with EcoRI endonuclease. Ten nanomolar labeled DNA, $80 \mathrm{nM}$ unlabeled DNA, and $1.6 \mathrm{nM} \mathrm{EcoRI} \mathrm{were} \mathrm{incubated} \mathrm{in} \mathrm{the} \mathrm{reaction} \mathrm{buffer} \mathrm{at}$ $27^{\circ} \mathrm{C}$. Dotted lines are the original data, which were fitted with $\mathrm{Eq}$. [2]. The fitted curves are represented by solid lines. During the reaction the cross-correlation amplitude $\mathrm{G}_{\mathrm{gr}}(0)$, which is a measure of reaction progress, gradually decreases. Reproduced with permission from U. Kettling, A. Koltermann, P. Schwille, and M. Eigen, (1998) Proc. Natl. Acad. Sci. USA 95, 1416-1420. Copyright 1998 National Academy of Sciences, U.S.A.

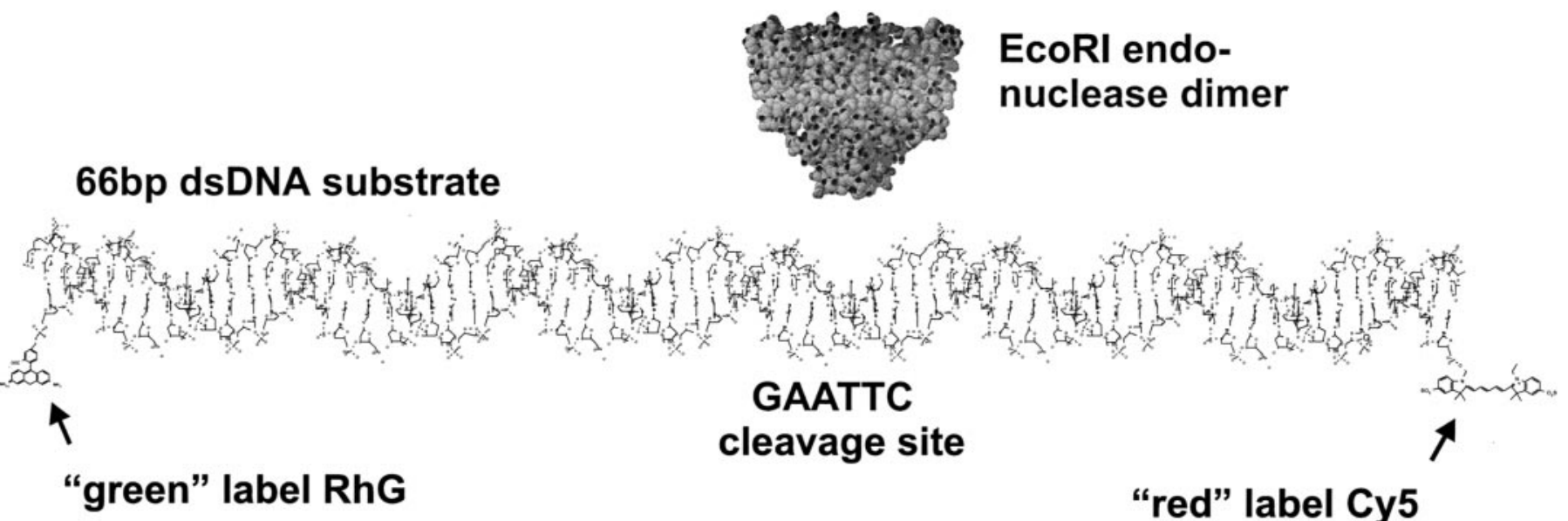

FIG. 4. Endonuclease EcoRI homodimer and RhG-Cy5-labeled 66-bp oligonucleotide structures drawn in scale. The labeled 5' ends of the DNA strands are well separated from the recognition site of the endonuclease and will therefore not interfere with the enzymatic process. Based on different molecule coordinates obtained from The Protein Data Bank, http://www.rcsb.org/pdb [34]. 
$130 \mathrm{nM}$ (Fig. 6). The results confirmed the well-known Michaelis-Menten behavior of EcoRI, revealing a $K_{m}$ of $14 \pm 1 \mathrm{nM}$ and a $\mathrm{k}_{\text {cat }}$ of $4.6 \pm 0.2 \mathrm{~min}^{-1}$. Comparison of these kinetic constants with literature data is difficult, because a variety of substrates have been used under different assay conditions, and especially $K_{m}$ values seem to depend sensitively on the type of substrate employed. Methods, however, that are, like off-line el ectrophoretic analyses of plasmid cleavage, as sensitive as dual-color FCS and enable kinetic analyses at nanomolar concentrations and below revealed similar $\mathrm{K}_{\mathrm{m}}$ values in the low nanomolar range (1-10 nM) (32). These values fit well with the natural function of restriction endonucleases, i.e., to recognize and cleave single copies of foreign DNA molecules in a bacterial cell (1 nM corresponds to approximately one molecule per Escherichia coli cell). Higher $\mathrm{K}_{\mathrm{m}}$ values obtained by other methods are supposed to be artifacts from measurements with artificial substrates at higher concentrations. This corresponds to the often addressed demand to analyze enzyme kinetics at reasonable substrate concentrations above and below the expected $\mathrm{K}_{\mathrm{m}}$.

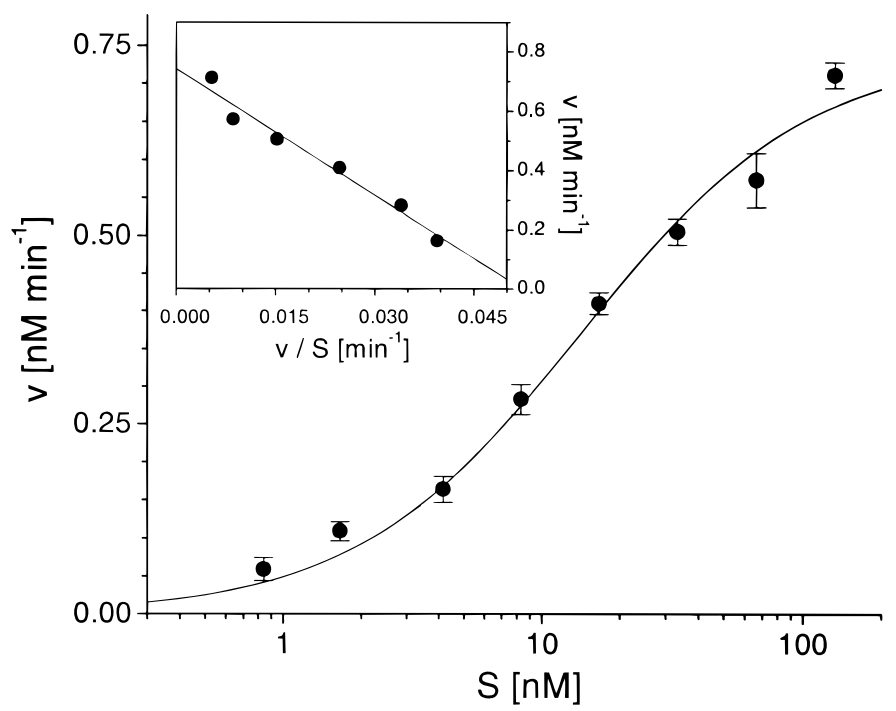

FIG. 6. Michaelis-Menten plot. Labeled DNA at a final concentration of $0.8 \mathrm{nM}$ was mixed with different concentrations, (0-130 nM) of unlabeled DNA, and incubated with $160 \mathrm{pM} E$ coRI in the reaction buffer at $27^{\circ} \mathrm{C}$. The reactions were monitored on-line and the initial rates $v$ were derived by linear regression of data points of the first 5-20 min. Inset: Calculations from an Eadie-H ofstee plot lead to a $\mathrm{K}_{\mathrm{m}}$ value of $14 \pm 1 \mathrm{nM}$ and $\mathrm{v}_{\max }$ of $0.74 \pm 0.03 \mathrm{nM} \mathrm{min}^{-1}$. Reproduced with permission from U. Kettling, A. Koltermann, P. Schwille, and M. Eigen, (1998) Proc. Natl. Acad. Sci. USA 95, 1416- 1420. Copyright 1998 National Academy of Sciences, U.S.A.

\section{OTHER APPLICATIONS}

Similar to the dissociation of two labeled fragments, the association of the labels can also be monitored by dual-color FCS. Rigler et al. (18) report the incorporation of labeled oligonucleotide primers into a PCR product. The forward and reverse primers of the reaction are labeled with different fluorescent dyes. Correlation of the emission of the dyes can be sensitively detected after incorporation into the resulting double-stranded PCR product. Koltermann et al. report the enzymatic degradation of a double-label ed peptide by an unspecific protease subtilisin (19). Besides unspecific proteases like subtilisin, sequence-specific proteases are of vital importance in numerous biological processes. There fore, the characteristics of a sequence-specific protease from tobacco etch virus (TEV protease) has been studied in the authors' laboratory using dual-color cross-correlation spectroscopy. TEV protease specifically recognizes and cleaves the amino acid sequence ENLYFQ(G/ S). The cleavage of a double-labeled peptide containing this recognition sequence is shown in Fig. 7. While the sample without addition of enzyme shows no degradation during the course of the measurement, TEV protease specifically degrades the peptide substrate. The reaction can be followed over the whole nanomolar concentration range. But not only enzymatic processes can be observed using dual-color approaches; protein-

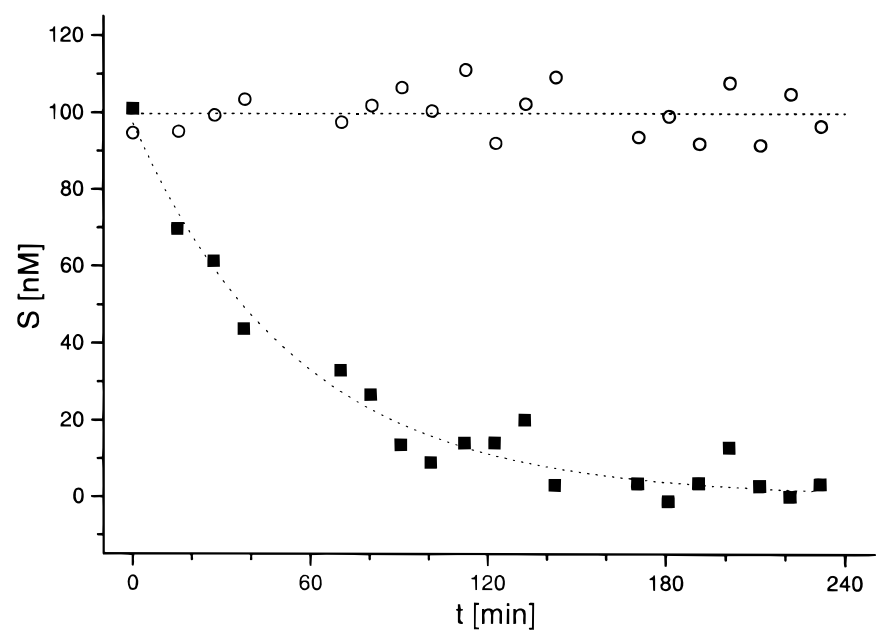

FIG. 7. Protease cleavage of a peptide substrate monitored by crosscorrelation FCS (U. Kettling and A. Koltermann, unpublished results). One hundred nanomolar double-labeled peptide (Alexa 488, Cy5) containing the cleavage site for tobacco etch virus protease is incubated with (filled squares) and without (open circles) $0.01 \mathrm{U} / \mu \mathrm{l}$ protease in assay buffer containing $50 \mathrm{mM}$ Tris- $\mathrm{HCl}, \mathrm{pH} 8.0,0.5$ mM EDTA, 10 mM DTT, 0.05\% glycerol. 
protein interactions can also be detected, as demonstrated only recently by Bieschke et al. (12) with the sensitive measurement of prion-protein aggregations. The homogenous assay proposed by Bieschke et al. specifically detects the presence of prion-protein aggre gates in spinal liquor. The method relies on the extreme sensitivity and specificity of dual-color fluorescence spectroscopy since these prion-protein aggregates are present in extremely low concentrations in the early phase of prion-connected diseases like Alzheimer's disease and Creutzfeldt-J akob disease.

\section{EXPERIMENTAL PROTOCOL}

The main prerequisite for measuring enzyme kinetics by dual-color FCS are substrates that are labeled with two spectrally distinguishable fluorophores. As a rule, the signal-to-noise ratio of cross-correlation analysis increases with the spectral separation of the two fluorophores employed. I deally, the fluorophores should have excitation and emission spectra that do not overlap at all, to avoid energy transfer as well as cross-talk between the two channels. In practice, however, there exists another rather simple but considerable constraint: the wavelengths of available laser lines. Usually the blue 488-nm line of an $\mathrm{Ar}^{+}$Iaser and the red 633-nm line of a HeNe laser are used as excitation light sources. With these wavelengths, a typical pair of fluorophores is R hodamine Green (RhG, Molecular Probes) as the green label and Cy5 (Amersham Pharmacia) as the red label. Their excitation and emission spectra, as shown in Fig. 2, fit well with the two laser lines. Moreover, the emission spectrum of RhG has only a small overlap with the excitation spectrum of Cy5, resulting in a low-energy transfer and cross-talk. Alternative green labels with only slightly different spectral characteristics are Oregon Green (alsoa rhodamine derivative, Molecular Probes), Alexa 488 (Molecular Probes), and Cy2 (Amersham Pharmacia); an alternative red label is BODIPY 650/665 (Molecular Probes). All of these fluorophores are available as reactive derivatives. On the other hand, the standard green dye that is excitable at $488 \mathrm{~nm}$, fluorescein, is not suited at all for FCS and other singlemolecule analyses due to its strong tendency toward photobleaching.

After proper choice of fluorophores these have to be attached to the substrate of interest. For DNA substrates, there are several ways to attach fluorophores specifically to a certain position in the molecule. The first and most simple way is to introduce the label during chemical synthesis of theoligonucl eotide. Some of the fluorophores are available as phosphoamidites and can be attached to the $5^{\prime}$ end of an oligonucleotide as the final step during solid-phase synthesis on a nucleic acid synthesizer. As an alternative, fluorophores can be attached to the oligonucleotide after synthesis. There exist several al ternative protocols for the chemical labeling of an oligonucleotide. The main constraint, however, lies in the availability of suitably modified fluorophore derivatives. The most prominent protocol attaches an amino linker at the $5^{\prime}$ end during synthesis, which can afterward be labeled with a fluorophore carrying an amino-reactive group such as an $\mathrm{N}$-hydroxysuccinimidyl ester. The third way to label oligonucleotides uses a polymerase to introduce the fluorescent labels into the DNA strand (11). The method takes advantage of the fact that several of the fluorophores are availableas nucleosidetriphosphates (either dNTPs or rNTPs). The labeling can be achieved simply by adding the fluorophore-labeled nucleoside triphosphates to a primer extension reaction, the result of which is directly the labeled nucleic acid molecule. While this labeling is primarily site unspecific, a modification enables the site-specific labeling of DNA double strands: after cutting a DNA strand at the ends with a restriction endonuclease that releases fragments with overhanging $5^{\prime}$ ends and purification of the DNA by gel electrophoresis, the overhang can be refilled with one or more fluorophore-labeled nucleoside triphosphates by use of a polymerase (typically the Klenow fragment). However, when unlabeled nucleoside triphosphates are added to the mixture to fill gaps, it is important to consider the much lower incorporation rate of dye-labeled nucleotides into DNA by polymerases. I n general, short DNA substrates should be label ed either during synthesis or afterward at certain functional groups such as amino groups. Longer DNA substrates should preferably be end-labeled via refilling appropriate $5^{\prime}$ overhangs or by using $5^{\prime}$ labeled primers and synthesizing the DNA molecule via PCR. Purification from excess reactive fluorophore or from excess labeled PCR primers should be done by standard reversed-phase HPLC or polyacrylamide gel el ectrophoresis to avoid fluorescent background in the assay. The degree of labeling (in a purified sample) can easily be determined by measuring the absorption at 260-nm for DNA and at the respective absorption maxima of the employed fluorophores.

The fundamentals of fluorescence correlation spectroscopy data analyses have been given in detail above. Correlation analysis is done at pretermined intervals (usually every 10-30 s) and the resulting correlation curves are fitted using Eq. [5]. Fitting is usually done with a Marquardt nonlinear least-squares regression routine implemented either into special FCS software 
such as the FCS Access package (EVOTEC BioSystems, Hamburg, Germany) or into standard data analysis software such as Microcal Origin (Microcal Software, Northampton, MA). Finally, concentrations of dual-col or molecules are calculated from Eq. [8] using the $\mathrm{G}(0)$ values from both the cross-correlation and two autocorrelation curves. The structure parameter $\mathrm{z}_{0} / \mathrm{r}_{0}$ is usually determined by evaluating the autocorrelation curves of pure Cy5 and Rhodamine Green solutions. The effective detection volume $V_{\text {eff }} \cong \pi^{3 / 2} r_{0}^{2} z_{0}$ has an ellipsoidal shape where $r_{0}$ and $z_{0}$ are the horizontal and vertical axes. $V_{\text {eff }}$ is cal culated from autocorrelation measurements with a pure dye solution, e.g., with a Rhodamine Green sol ution (diffusion coefficient $\mathrm{D}_{\mathrm{RhG}}=2.8 \times 10^{-10} \mathrm{~m}^{2} \mathrm{~s}^{-1}$ ) using the relationship $r_{0}=\left(4 D \tau_{\text {Diff }}\right)^{1 / 2}$ (E q. [7]). Typical volumes are in the range 0.1 to $10 \mathrm{fl}$. Thediffusion time of the pure doublelabeled substrate can be measured in a reference sample without enzyme. Parameters $z_{0} / r_{0}$ and $\tau_{\text {Diff,DNA }}$ then are fixed during the analysis of the correlation curves. The concentration value at $t_{\infty}$, which is usually obtained from a measurement with excess enzyme and that is in most cases due to an unspecific detector cross-talk (17), can be subtracted as an offset; the resulting values must then be normalized to the initial substrate concentrations. Finally, the evaluation of kinetic constants, such as $K_{m}$ and $k_{c a t}$, is done via a Michaelis-Menten plot as is known from the literature.

\section{CONCLUDING REMARKS}

Dual-color fluorescence spectroscopy provides a sensitive and convenient means to observe biochemical interactions and reactions at the single-molecule level in very small sample volumes. Dual-color methods have proven their potential to monitor the kinetics of endonuclease, polymerase, and protease with high sensitivity and selectivity in real time. Confocal microscopes for dual-col or fluorescence spectroscopy are now becoming commercially available. These system are much more versatileand convenient to handlethan the first experimental setups. In parallel, new and promising photophysical methods and powerful algorithms to evaluate fluorescence fluctuation data have been developed or are under investigation. Therefore, new and fascinating applications of dual-color methods in biochemistry as well as in other fields of the life sciences are expected in the near future.

\section{ACKNOWLEDGMENTS}

The authors are grateful to Dr. Petra Schwille and Katrin Heinze for stimulating discussions. This work was supported by the German Bundesministerium für Bildung, Wissenschaft, Forschung und Technologie (Grant FK 0310739 A) and by the Volkswagen Stiftung (Grant $113 a / 6.8 .2 .3)$.

\section{REFERENCES}

1. Magde, D., Elson, E. L., and Webb, W. W. (1972) Phys. Rev. Lett. 29, 705-708.

2. Elson, E. L., Magde, D., and Webb, W. W. (1974) Biopolymers 13, 29-61.

3. Elson, E. L., and Magde, D. (1974) Biopolymers 13, 1-27.

4. Ehrenberg, M., and Rigler, R. (1976) Q. Rev. Biophys. 9, 69-81.

5. Ehrenberg, M., and Rigler, R. (1974) Chem. Phys. 4, 390- 401.

6. Widengren, J ., Rigler, R., and Mets, U. (1998) Fluorescence 4, 255- 258.

7. Schwille, P., Kummer, S., Heikal, A. A., Moerner, W. E., and Webb, W. W. (2000) Proc. Natl. Acad. Sci. USA 97, 151-156.

8. Widengren, J., Mets, U., and Rigler, R. (1999) Chem. Phys. 250, 171- 186.

9. Edman, L., Foldespapp, Z., Wennmalm, S., and Rigler, R. (1999) Chem. Phys. 247, 11-22.

10. Wennmalm, S., Edman, L., and Rigler, R. (1997) Proc. Natl. Acad. Sci. USA 94, 10641- 10646.

11. Schwille, P., Oehlenschläger, F., and Walter, N. G. (1996) Biochemistry 35, 10182-10193.

12. Bieschke, J ., Giese, A., Schulz-Schaeffer, W., Zerr, I., Poser, S., Eigen, M., and Kretzschmar, H. (2000) Proc. Natl. Acad. Sci. USA 97, 5468-5473.

13. Kettling, U., Koltermann, A., Schwille, P., and Eigen, M. (1998) Proc. Natl. Acad. Sci. USA 95, 1416- 1420.

14. Nishimura, G., Rigler, R., and Kinjo, M. (1997) Bioimaging 5, 129-133.

15. Kinjo, M., Nishimura, G., Koyama, T., Mets, U., and Rigler, R. (1998) Anal. Biochem. 260, 166- 172.

16. Eigen, M., and Rigler, R. (1994) Proc. Natl. Acad. Sci. USA 91, 5740-5747.

17. Schwille, P., Meyeralmes, F. J ., and Rigler, R. (1997) Biophys. J . 72, 1878-1886.

18. Rigler, R., Foldespapp, Z., Meyeralmes, F.J ., Sammet, C., Volcker, M., and Schnetz, A. (1998) J . Biotechnol. 63, 97- 109.

19. Koltermann, A., Kettling, U., Stephan, J ., Winkler, T., and Eigen, M. (2000) in Fluorescence Correlation Spectroscopy: Theory and Application (Rigler, R., and Elson, E., Eds.), Springer-Verlag, Heidelberg, in press

20. Winkler, T., Kettling, U., Koltermann, A., and Eigen, M. (1999) Proc. Natl. Acad. Sci. USA 96, 1375- 1378.

21. Heinze, K. G., Koltermann, A., and Schwille, P. (2000) Proc. Natl. Acad. Sci. USA 97, 10377-10382.

22. Kask, P., Palo, K., Ullmann, D., and Gall, K. (1999) Proc. Natl. Acad. Sci. USA 96, 13756-13761.

23. Chen, Y., Muller, J . D., So, P. T. C., and Gratton, E. (1999) Biophys. J. 77, 553-567.

24. Kask, P., Palo, K., Fay, N., Brand, L., Mets, U., Ullmann, D., J ungmann, J., Pschorr, J ., and Gall, K. (2000) Biophys. J. 78, 1703-1713. 
25. Schwille, P. (2000) in Fluorescence Correlation Spectroscopy: Theory and Applications (Rigler, R., and Elson, E., Eds.), Springer-Verlag, Heidelberg, in press.

26. Xu, C., Zipfel, W., Shear, J . B., Williams, R. M., and Webb, W. W. (1996) Proc. Natl. Acad. Sci. USA 93, 10763-10768.

27. Shear, J . B., Brown, E. B., and Webb, W. W. (1996) Anal. Chem. 68, 1778-1783.

28. Denk, W., Strickler, J. H., and Webb, W. W. (1990) Science 248, 73-76.

29. Aragon, S. R., and Pecora, R. (1976) J. Chem. Phys. 64, 1791-1803.
30. Waters, T. R., and Connolly, B. A. (1992) Anal. Biochem. 204, 204-209.

31. Ghosh, S. S., Eis, P. S., Blumeyer, K., Fearon, K., and Millar, D. P. (1994) Nucleic Acids Res. 22, 3155- 3159.

32. Halford, S. E., andJ ohnson, N. P. (1981) Biochem.J . 199, 767-777.

33. Hedgpeth, J., Goodman, H. M., and Boyer, H. W. (1972) Proc. Natl. Acad. Sci. USA 69, 3448-3452.

34. Berman, H. M., Westbrook, J ., Feng, Z., Gilliland, G., Bhat, T. N., Weissig, H., Shindyalov, I. N., and Bourne, P. E. (2000) Nudeic Acids Res. 28, 235-242 